Table VII.-Constants of a Few Essential Oils. Sp. gr. at $\mathrm{r}^{\circ}$. Ventzke.

Polatization
I00 $\mathrm{mm}$

oil.

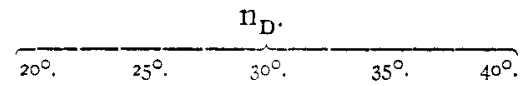

A large number of essential oils have been examined during the course of drug inspection of this department, and the following list gives the maximum variation of the refraction from readings in Table VII of those oils found by other tests to have been pure.

TABLE VIII.

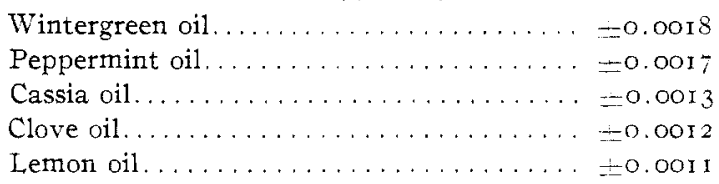

A sufficient number of the other oils of known purity have not as yet been examined to state the variations in refraction, but at least two reliable samples of each oil obtained from different sources have been tested for refraction with closely agreeing results.

\title{
THE DETECTION OF METHYL ALCOHOL. ${ }^{3}$
}

\author{
By HeYwaRd SCUDDER. \\ Received April 24, 1905.
}

THE TESTS for the detection of methyl alcohol are used chiefly to distinguish it from ethyl alcohol, so their value is dependent first on a sharp distinction from ethyl alcohol, then on their delicacy and rapidity. In the following description the standard of delicacy

1 Readings are all averages from a large number of samples.

2 Made from the sweet birch.

${ }^{8}$ Presented before the New York Section of the American Chemical society in February, 1905 . 
will be taken as the quantity of ethyl alcohol that can be detected in ethyl alcohol. Most of the tests will show very much smaller quantities of methyl alcohol in water, but their use for such a purpose is practically unknown.

ODOR TESTS.

This is made by adding to the solution, salicylic acid and concentrated sulphuric acid and warming, thus forming methyl salicylate which is supposed to have a characteristic odor (of oil of wintergreen). In experiments made on eight persons $I$ found that none of them could distinguish between ethyl and methyl alcohol by this test, because the odor of ethyl salicylate so strongly resembles that of methyl salicylate. If told that both alcohols were present a difference in odor was often noticed, but if solutions of unknown content were given, $e . g$., two tubes of methyl or two of ethyl alcohol, the test failed even when comparative tests with the alcohols were made.

All tests dependent on odor are unsatisfactory because it is impossible to make accurate comparative tests. After smelling any odoriferous body, probably on account of persistence of the odor in the nasal passages, or possibly from slow reactivity of the olfactory nerves, it is not possible for an ordinary person to have an accurate perception of the odor of a second body. This can readily be shown experimentally, most surprising mistakes being made after the sense of smell is once blunted. In making color tests the comparison is direct and the subjective effect of memory is eliminated.

I have been told that this test has been used in trials, for adulteration of liquors, to show juries the difference between the two alcohols, the point being made that there is a difference in the rapidity of the production of the odor as well as in the quality. I consider the test unreliable and not sufficiently rapid to be an improvement on the test by oxidation with a hot copper spiral and testing for the oxidation product by resorcin. The difference in color between the condensation products of acetaldehyde and resorcin and formaldehyde and resorcin is great enough to be appreciated by any juror.

\section{COLOR TESTS.}

In the Riche and Bardy ${ }^{1}$ test the methyl alcohol is converted

1 Compt. Rend., 80, 1076 (1875). 
into methyl aniline which is then tested for. The test is too long and slow to be of much use to-day.

In all the other tests the alcohol is oxidized to formaldehyde, methylal or formic acid. The color test is then applied to this oxidation product. Methylal gives the same reaction as formaldehyde in almost all tests. If ethyl alcohol is present, either the color test must be given only by the oxidation product of methyl alcohol-as in oxidation to the acid, formic acid giving a dark color with silver nitrate while acetic acid gives no color-or enough of the corresponding oxidation product of ethyl alcohol must be removed to prevent interference with the color. The difficulty of fulfilling these conditions is the chief cause of failure in the tests used. It is practically impossible to control oxidation so as to get a good yield of formic acid and the tests for its detection are not very delicate. The removal of all the acetaldehyde without some loss of formaldehyde is impossible, but this method is perfectly practicable since the tests for formaldehyde are extremely delicate. The fact that pure ethyl alcohol, when oxidized by any of the methods used in the tests for methyl alcohol, gives some formaldehyde is not sufficiently considered. It has been shown ${ }^{1}$ that when ethyl alcohol is oxidized by a heated copper spiral, formaldehyde is formed in small amounts. I have found that this is also true when ethyl alcohol is oxidized in cold dilute solution by potassium bichromate and sulphuric acid, or by potassium permanganate and sulphuric acid, enough formaldehyde being made to give the gallic acid test.

Therefore, it is obvious that the test used to show the presence of formaldehyde, after oxidation of the alcohol, must not be too delicate or it will apparently show the presence of methyl alcohol when none is really there. This is the defect of the gallic acid reaction ${ }^{2}$, an extremely characteristic test for formaldehyde and one little obscured by the presence of other compounds, but so delicate that it is given by solutions of ethyl alcohol, cane-sugar and a great variety of compounds after oxidation by a heated copper spiral ${ }^{3}$.

The tests that follow are grouped by the reagent used for the

1 Mulliken, scudder : Am. Chem. $J$, 24, 446 (1900).

2 The solution to be tested is mixed with $0.2 \mathrm{cc}$. of a saturated alcoholic solution of gallic acid and poured on top of (not mixed with) concentrated sulphuric acid in a testtube. A contact ring of blue or green and blue shows the presence of formaldehyde.

3 Mulliken, Brown and French: $A m$. Chem. J., 25, ris (1904). 
production of the color, in order to make more clear a general understanding of the difficulties and of the sources of error.

REAGENT, SILVER NITRATE.

Miller Test. ${ }^{1}$ - This consists in oxidation in the cold by potassium bichromate and sulphuric acid, distillation and testing the distillate for formic acid by silver nitrate. Allyl alcohol and acetone are said to give the same reaction. It is noted that a trace of formic acid or some other reducing agent is formed from ethyl alcohol. The test is not delicate and is of little importance.

REAGENT, LEAD PEROXIDE.

Trillat Test. ${ }^{2}$ - This consists in oxidation in cold dilute solution by potassium bichromate and sulphuric acid, under which conditions methyl alcohol is said to give methylal, ethyl alcohol to give acetaldehyde, acetal and acetic acid. The acetaldehyde is removed by distilling $25 \mathrm{cc}$. The next roo cc. distilled contain the methylal. One-half of this is digested in a closed flask with dimethylaniline at $70-80^{\circ}$ for three hours, forming tetramethyldiaminodiphenylmethane. The solution is made alkaline and the excess of the dimethylaniline distilled. The residue is acidified by acetic acid and a little lead peroxide added. On boiling, a blue color appears, if methyl alcohol is present. It is said to show 0.2 per cent. methyl alcohol in ethyl. The time required for the test is about five hours.

The difficulties are numerous. If all the acetaldehyde is not removed by the fractional distillation, what remains may give a color that resembles or interferes with that from methylal. Since some methylal distils over with the acetaldehyde it is not safe to distil off much more than $25 \mathrm{cc}$. to remove the latter, for if only a small amount of methyl alcohol is present, the loss might be sufficient to spoil the test. The dimethylaniline and the lead peroxide must be very pure or the color may be affected. In distilling off the excess of dimethylaniline (this procedure is necessary because dimethylaniline gives a violet or blue color with lead peroxide) some methylal is lost as may be shown by tests. I have found it easy to be sure of the removal of all the dimethylaniline, by filtering through a wet filter before distilling. No special care is necessary in

1 Allen's "Commercial Orgarric Analysis," Vol. I, p. 8r (3d Ed.).

2Ann. Chim. Anal. Appl., 4, 42 (1899); Compt. Rend., 128, 438 (1899); Bull. Soc. Chim. (3) 21, 145 ( 1899 ). 
acidifying after the removal of the dimethylaniline, since a great excess of acetic acid has no effect on the color. The amount of lead peroxide used is actually a variable quantity. The directions call for four to five drops of a suspension in water. In addition to the variable strength of different samples, another error is possible here, since the amount of lead peroxide present will depend on how thoroughly the suspension is shaken before use. If too little is used, the color is faint. If too much is used, the color changes from blue to brown, though the effect of heat in deepening the color still persists.

The depth of color is supposed to be proportional to the amount of methyl alcohol originally present, but it is sometimes difficult to get this result. I have found a much fainter color from a solution that contained io per cent. of methyl alcohol than from one that contained I per cent., though the procedure was the same in each case.

I believe that the development or deepening of the color by boiling is characteristic. Acetaldehyde, after digestion with dimethylaniline and subsequent treatment as in the Trillat test, gives in the cold a color varying from green-yellow to green-blue. Dimethylaniline in the cold gives with lead peroxide a color varying from green-yellow to pure blue, but in both cases the color does not deepen on boiling, while the blue from methyl alcohol develops only on boiling. The color may be diminished or changed by the use of the wrong amount of lead peroxide, but here again boiling makes a characteristic deepening, if methyl alcohol was in the original solution tested, so that if there is a color in the cold that disappears or is not deepened by boiling, no methyl alcohol is present (provided the test has gone well). If the color deepens on boiling, even if it is not a pure blue but possibly a green-blue or brown, methyl alcohol is present.

Wolf ${ }^{1}$ states that pure ethyl alcohol gives the same reaction as methyl when the Trillat test is used, but that if the digestion with dimethylaniline is made at room temperature for twenty-four hours instead of at $70-80^{\circ}$ for three hours the test works perfectly. He claims that o. I per cent. of methyl alcohol can be shown in ethyl alcohol in this way. Since he makes no statement of the effect of heat on the color, the value of the improvement is doubtful, for it is possible that the color obtained from ethyl 
alcohol was due to the incomplete removal of acetaldehyde. Robine $^{1}$ gives an elaborate criticism of the Trillat test, and suggests several small changes in the procedure. His most essential points are the recommendation to make a blank test of every fresh sample of dimethylaniline; the necessity for having pure lead peroxide and for using the proper amount; the difficulty of removing all the acetaldehyde without loss of methylal and the fact that the test is spoiled unless all the acetaldehyde is removed. The presence of acetaldehyde is sometimes indicated by a yellow color of the solution and a precipitate of aldehyde resin after the excess of dimethylaniline has been distilled off. His criterion for the presence of methyl alcohol is that the final test must give a pure blue appearing hot, disappearing cold. Any shade of blue that has green in it, green-yellow or pure green is not to be regarded, since it may come from incomplete removal of acetaldehyde.

Since Robine notes the effect of heat on the color, it would appear probable that he tried heating in the cases where the color was due to acetaldehyde, but the fact that in such cases no deepening occurs when the solution is boiled is not mentioned by him. This differs from my observations previously given.

It is evident that the Trillat test must be used with care. It is delicate and fairly rapid, but the test may be spoiled not only by slight variations in the procedure, but also by causes of unknown origin which occur when the procedure is strictly followed. It is certainly not a test to be recommended to persons of limited knowledge of chemistry.

\section{REAGENT, RESORCIN.}

Mulliken-Scudder ${ }^{2}$ Test.-In its simplest form this consists in oxidizing a dilute solution of the alcohol by plunging into it several times a spiral of copper wire heated to redness. The formaldehyde formed is tested for by adding one drop of a 0.5 per cent. aqueous solution of resorcin and pouring, without mixing, on top of a little concentrated sulphuric acid in a test-tube. A contact ring and, on very gentle shaking, flocks of a characteristic red color appear, if formaldehyde is present. Acetaldehyde gives a ring and flocks of a color that varies from yellow to brown. This

1 Ann. Chim. Anal. Appl, 6, 128, 17I (I90I); Cf. Euler, Ber.. 37, 34II (1904), and Bach, Ibid., 37, 3986 (I904), on the unreliability of the Trillat test for formaldehyde, which is the same as the methyl alcohol test beginning with the digestion with dimethylaniline.

2 Mulliken, Scudder : Am. Chem. J., 21, 266 (1899). 
shows 8-io per cent. of methyl alcohol in ethyl. The time required is about five minutes.

The modified form ${ }^{1}$ is more delicate. The oxidation is carried out in the same way, but the acetaldehyde is removed by boiling the solution down about one-half. This may be done over a free flame for rapid work. In boiling down, some formaldehyde is always lost, and the more rapid the boiling the greater the loss. Therefore, it is advisable to boil down at $25-30^{\circ}$ under diminished pressure in order to get the best results. Where little formaldehyde is present the formation of flocks is slow, so in this modified form after the solution is brought in contact with the sulphuric acid it must be allowed to stand for three minutes, then shaken very gently for a minute, causing the two liquid surfaces in contact to mix, but not allowing rapid or excessive mixing. If only a very little formaldehyde is present, rapid mixing prevents the formation of flocks, although it has little or no bad effect when much formaldehyde is present. The formation of flocks is a necessary part of the test, a contact ring of the proper color not being considered sufficient evidence, since acetone and dimethylethylcarbinol both give red contact rings. Methyl esters and ethers, methylal and secondary and tertiary butyl alcohols give the same reaction as methyl alcohol in this test. This would indicate that whenever a methyl group and oxygen are close together and not bound firmly to the remainder of a compound, oxidation may produce formaldehyde, the amount formed probably being dependent on the relative attraction between the different groups in the compound. This test shows 2 to 3 per cent. methyl alcohol in ethyl. The time required is about twenty minutes. One great advantage of the test is that the formation of flocks of characteristic color gives a very positive indication, much more so than the production of a contact ring or of a temporary color.

United States Pharmacopoeia Test.-Sadtler ${ }^{2}$ states that the committee for the revision of the Pharmacopeia found resorcin more delicate than phloroglucin as a test for the formaldehyde produced in the oxidation of methyl alcohol. They give the following directions. Ten cc. of the solution to be tested, having a concentration of about ro per cent. (in testing liquors or alcohol, the proper dilution must be made), are placed in a test-tube

I Mulliken, Scudder : Kbia., 24, 444 (1900).

2. Am.J. Pharm., 77, 106 (1905). 
surrounded by cold water. It is oxidized by plunging a red hot spiral of copper wire-of specified dimensions-to the bottom of the test-tube and holding it there one to two seconds. This is repeated five to six times. The filtered solution is boiled gently till any odor of acetaldehyde ceases to be clearly distinguishable. To the cooled solution one drop of a $I: 200$ aqueous solution of resorcin is added and the mixture is floated on top of concentrated sulphuric acid in a test-tube. After standing three minutes the tube is gently rotated. If no rose-red ring appears, less than 2 per cent. of methyl alcohol is present. The time required for the test is about fifteen minutes. ${ }^{1}$

Mulliken Test ${ }^{2}$.-The alcohol is oxidized by a heated copper spiral, ammonia is then added and the solution is boiled gently in a dish till the odor of ammonia has disappeared. The acetaldehyde is thus driven off and the formaldehyde is converted into hexamethylentetramine. Concentrated hydrochloric acid is then added and the solution brought momentarily to boiling to decompose the hexamethylentetramine. The formaldehyde set free is tested for by resorcin as in the Mulliken-Scudder test. The test shows so per cent. methyl alcohol in ethyl, and an expert is said to be able to detect less than this amount.

This test is no more delicate than the simple form of the MullikenScudder test so that its advantage is not apparent. Theoretically, the method is one that promises well. Ammonia unites with formaldehyde very rapidly to form hexamethylentetramine, a compound that is stable on boiling and does not distil off to any extent, while acetaldehyde-ammonia is decomposed by heat and the acetaldehyde can be completely driven off by boiling. There are two defects in the process that I have not been able to overcome in any way. The addition of ammonia retains all the formaldehyde produced in the oxidation of ethyl alcohol, so that any delicate test applied after the decomposition of the hexamethylentetramine, whether the test is for the formaldehyde or for the ammonia, is given both by ethyl and methyl alcohol.

1 When I read this paper, I gave the outlines of a rapid test that would show 2 per cent. methyl alcohol in ethyl. Since that time Sadtler has publisited the test just given. The procedure of my test differed in several points, but the two are so much alike that it seems inadvisable to burden the literature with the details of mine. The fact that with such differences in procedure the results obtained are the same, shows that the pharmacopoeia test can be recommended to persons of limited chemical experience.

2 Provisional Methods for A nalysis of Foods (U. S. Dept. Agriculture, Bureau Chemistry, Bull. 65, 73 (1902). 
The oxidation by a copper spiral does not produce enough formaldehyde from methyl alcohol to allow as a test the use of the formation of some compound of hexamethylentetramine, such as the tetraiodide or the mercuric chloride compound, although both these compounds (which give difficultly soluble precipitates) can be used to detect the presence of quite small quantities of hexamethylentetramine. No precipitate is formed from quantities of hexamethylentetramine sufficient to give, after decomposition, fairly thick flocks in the resorcin test. In this decomposition some formaldehyde is always lost, since other compounds besides ammonia and formaldehyde are formed. It is advisable to boil for fifteen or twenty seconds or longer to get the maximum amount of formaldehyde.

\section{REAGENT, FUCHSINE.}

Another use of hexamethylentetramine gave promise but fails at times for reasons that I cannot discover. The solution is oxidized by a heated copper spiral, filtered, an excess of ammonia added and then boiled down about two-thirds. It is then cooled and $2 \mathrm{cc}$. of fuchsine aldehyde reagent added. The sulphurous acid in this decomposes the hexamethylentetramine and the aldehyde set free instantly colors the solution. A blank of pure water and fuchsine reagent is made, and the difference in color and the time noted. In this way it was possible to show I per cent. methyl alcohol in ethyl in fifteen minutes. But at times pure ethyl alcohol would give a color as deep and as rapidly formed as that from methyl alcohol. As a rule ethyl alcohol when oxidized gives a color that is very faint at first and does not deepen for so long a time that the distinction between it and that from methyl alcohol is sufficiently marked to make a good test.

REAGENT, PHLOROGLUCIN.

Acetaldehyde in amounts less than o.or per cent. gives no reaction with an alkaline solution of phloroglucin in the Haigh and the Prescott tests, but will give a reaction with I.o cc. or $2.5 \mathrm{cc}$. of alkaline phloroglucin as used in the Sangle-Ferriére test. In large quantities-over o.I per cent.-it will also give a color with the phloroglucin solution in the Haigh test. Formaldehyde in very small amounts gives a color permanent for several minutes. The color is said to be pure red. Actually it depends on the relative concentrations of the formaldehyde and the phloroglucin, varying 
from yellowish red through pure red to violet-red. Since, in any solution to be tested, the quantity of methyl alcohol present may be anywhere from I per cent. to roo per cent., oxidation will produce a very variable amount of formaldehyde, but the conditions of any test call for a fixed amount of phloroglucin; so the color may be pure red or may be any of the shades mentioned.

Sufficient formaldehyde is formed in the oxidation of ethyl alcohol to give a color with phloroglucin, though this color is fainter than that obtained from the oxidation of solutions containing over ro per cent. of methyl alcohol. The color is said to differ in quality from that obtained from oxidized methyl alcohol. I have not found this statement to be true, for the reason just given.

The color obtained from oxidized methyl alcohol is said to be much more permanent than that given by oxidized ethyl alcohol. While this is true, the color given by ethyl alcohol often lasts for a number of minutes, so that it is impossible to distinguish between ethyl and methyl alcohol either by this criterion or by the difference in color, without making a comparative test with ethyl alcohol. In some experiments on the duration of the color (the oxidant was a heated copper spiral), a ro per cent. aqueous solution of methyl alcohol gave a red that lasted for ten minutes without any noticeable fading; then it gradually grew lighter. At the end of twenty minutes the color was still well marked. At the end of one hour there was no color. A 20 per cent. aqueous solution of ethyl alcohol gave a distinct red that lasted for six minutes, then began to fade. At the end of fifteen minutes the color was very faint. A 20 per cent. aqueous solution of a mixture of 2 parts methyl alcohol and 98 parts ethyl alcohol, boiled down onehalf after oxidation, gave a red that was deep for one minute, then faded but was noticeable for more than ten minutes. A 20 per cent. solution of ethyl alcohol similarly treated gave a color that faded from the beginning and was hardly noticeable after three minutes.

The full depth of color is sometimes not reached for one to two minutes. The duration of the color is lessened by stirring.

When the solution is boiled, the color changes to a clear brownyellow. This same color develops on boiling solutions that have become colorless. When cooled, after boiling, the color from methyl alcohol is permanent for one to two minutes, then fades 
more or less slowly, while the color from ethyl alcohol becomes very faint or disappears as soon as the solution is cold. This disappearance or fading of color in the case of ethyl alcohol is noticed with certainty only when an excess of alkali is added, since the phloroglucin solution as ordinarily prepared will give, after boiling, a faint violet in the cold. The excess of alkali destroys this violet color. When there is doubt as to the presence of methyl alcohol, on account of the faint or transient color obtained by the ordinary test with phloroglucin in the cold, the question can often be settled definitely by the addition of an excess of alkali, boiling and noting the depth and permanence of the color of the cold solution.

Whenever phloroglucin is used as the agent to detect formaldehyde in testing for methyl alcohol, a comparative test with pure ethyl alcohol must always be made, since it is impossible to state definitely the exact color or the exact duration of the color that will be obtained from varying quantities of methyl alcohol. In making this comparative test each solution must be added to the phloroglucin solution at the same time, so that the intensity and permanence of the colors may be judged by direct comparison.

Prescott Test. ${ }^{1}$ - In this test the alcohol is oxidized by a heated copper spiral, hydrogen peroxide is added to destroy the acetaldehyde, the excess of hydrogen peroxide is removed by sodium thiosulphate and the formaldehyde tested for by phloroglucin. This shows 5 per cent. methyl alcohol in ethyl. It is difficult to get correct results with this test, because the action of the hydrogen peroxide and the thiosulphate seem to go wrong at times. The Haigh test is much more satisfactory.

Haigh Test. ${ }^{2}$ - In this test the alcohol is oxidized by a heated copper spiral, the acetaldehyde removed by boiling the filtered solution till little or no odor is left and the phloroglucin test used to show the presence of formaldehyde. The color from methyl alcohol is said to last two to three minutes, that from ethyl alcohol to be faint and quickly to disappear. This shows 5 per cent. methyl alcohol in ethyl. The time required, including the blank of ethyl alcohol, is about fifteen minutes.

The test is as satisfactory as any that uses phloroglucin. By adding alkali and heating the solution after it has been mixed

1 Pharm. Archiv., 4, 86 (rgor).

2 Pharm. Review, 21, 404 (1903). 
with the phloroglucin (as previously described) it is possible to show 3 per cent. to 4 per cent. methyl alcohol by this test. The fact noted by Haigh that after one-half hour to one hour the solution from ethyl alcohol gives a blue color, is due in my opinion to the phloroglucin alone (possibly the color comes from some oxidation reaction) and has nothing to do with ethyl alcohol or its oxidation products.

Since acetaldehyde gives no color with alkaline phloroglucin, the question naturally arises, what is the reason for its removal in the Prescott and the Haigh tests? The answer is very simplethere is no need of removal. The working of the test has been misunderstood. When ethyl alcohol is oxidized, sufficient formaldehyde is produced to give a fairly deep and permanent color with alkaline phloroglucin solution, which is quite a delicate reagent for the detection of formaldehyde. The processes employed for the removal of acetaldehyde remove at the same time so much formaldehyde that the reaction with phloroglucin is reduced to a minimum. When methyl alcohol is present, sufficient formaldehyde is left to give a well-marked reaction. The experiments on the duration of color, given under the general treatment of the phloroglucin reaction, show this very clearly.

Sanglé-Ferriére-Cumiasse Test. ${ }^{1}$-This consists in oxidation of a cold, dilute solution by potassium permanganate and sulphuric acid. The excess of permanganate is reduced by tannin, the solution is made faintly alkaline and the manganese dioxide filtered off. The filtrate is tested by alkaline phloroglucin. It is said to show I per cent. methyl alcohol in ethyl.

The test has a simple procedure, but the length of time necessary to oxidize the alcohol in order to get satisfactory results seems to vary with the room temperature, the amount of alcohol present and the method of adding the sulphuric acid. The recommendation is made to confirm the test by acidifying and testing with gallic acid. This confirmation I regard as valueless, because of the great delicacy of the gallic acid test for formaldehyde. Ethyl alcohol often gives with phloroglucin quite a deep color, not the faint color mentioned in the description of the test.

CHOICE OF TESTS.

This will vary with circumstances. Unless there is supposed 1. Ann. Chim. Anal, Appl, 8, 82 (r903). 
to be only a very small amount of methyl alcohol present, I should recommend trying first the simple Mulliken-Scudder test which shows 8 per cent. to ro per cent and takes but a few minutes; next the Pharmacopeia test which shows 2 per cent. and takes fifteen minutes. If that gave no indication at all, the Trillat test should be used, but if there was any indication of the possible presence of methyl alcohol or a suspicion that it was there, I should advise rather than use even the Trillat test to concentrate by distillation through a Hempel tube or Le Bel-Henninger tube or some such device and then to use one of the shorter tests. This can be done much more quickly and easily than most persons suppose. In experiments to test the efficiency of the process when only small amounts of liquid are available, I used $25 \mathrm{cc}$. of a mixture of I per cent. methyl alcohol and 99 per cent. ethyl alcohol. This was fractionated through a Hempel tube made of glass tube $22.5 \mathrm{~cm}$. long, $1.7 \mathrm{cc}$, in diameter filled with glass beads about $3 \mathrm{~mm}$. in diameter. The beads held about $3^{\frac{1}{2}} \mathrm{cc}$. of liquid that would not drain back into the flask. Three fractionations were made, taking the first time I $5 \mathrm{cc}$, then $7 \frac{1}{2} \mathrm{cc}$. and finally $2 \mathrm{cc}$. One $\mathrm{cc}$. of this last fraction was diluted, oxidized by a heated copper spiral, boiled down one-half over a free flame and tested by resorcin. Moderately thick red flocks appeared. The total time consumed was one hour (this includes the time taken in allowing the liquid to drain back into the flask, a procedure necessary when such small quantities of liquid are being fractionated in this way). The Trillat test would have taken five hours. The Sanglé-Ferriére-Cumiasse test is claimed to have a delicacy sufficient to show this amount of methyl alcohol, but I am doubtful about its indications. None of the other tests is sufficiently delicate to use in such a case.

The great value of the resorcin test is in the positive evidence given and the advantage of having some idea in a rough way of the quantity of formaldehyde present. The formation of flocks of characteristic color shows positively the presence of a certain amount of formaldehyde and no comparative test with ethyl alcohol is necessary. If there is only a contact ring and no flocks, there is very little formaldehyde present. When more is present there will be scanty, small flocks. When much is present, the flocks are thick and abundant. 


\section{MIXTURES.}

In consequence of the great number of organic compounds, it is impossible to devise a test that will show a particular compound in any possible mixture. Certain general principles can be given, and by the aid of these and by a clear understanding of what the test used will show and what are its possibilities of error, the detection of methyl alcohol ought to be certain except when it is present in very small quantities and there is not sufficient material to allow of concentration.

If formaldehyde is present, it must be removed, if any of the oxidation tests are used, since they are all dependent on conversion of the alcohol to formaldehyde or methyla1. This may be accomplished by digestion with ammonia; by digestion cold with excess of sodium bisulphite for four to five hours ${ }^{1}$; by digestion at $70-80^{\circ}$ in a stoppered bottle with resorcin and sulphuric acid for two to three hours; ${ }^{2}$ or by boiling with a return condenser with aniline and phosphoric acid. ${ }^{3}$ The liquid is then distilled, the formaldehyde remaining behind in combination. The distillate should always be tested for aldehyde to make sure that the removal is complete. Other means that may prove useful in certain cases are digestion hot with sodium sulphite ${ }^{4}$ or wth hydrazine sulphate. ${ }^{5}$ When difficulty is found in removing all the formaldehyde, it is often possible without further treatment to make a rapid test which either will show the presence of methyl alcohol or will make further investigation desirable. Under these conditions little formaldehyde will be present and the resorcin test used before oxidation will show only a contact ring. When the solution is oxidized and the resorcin test again applied, if methyl alcohol is present, the increased amount of formaldehyde will be shown either by the presence of flocks or by thickening of the ring. The presence of flocks in such a case would be almost conclusive evidence of the presence of methyl alcohol. Fractional distillation is often of great help in such cases. Gnehm and Kaufler ${ }^{6}$ state that in the estimation of methyl alcohol in formaldehyde, when the formaldehyde is condensed with sodium sulphanilate

1 Bamberger: $Z$. angew. Chem, 17, 1246 (1904).

2 Mulliken, Scudder: $A$ m. Chem.J., 24, 449 (1900).

Allen, Chattaway : Analyst, 16, ir3 (1891).

4 Lemme: Chem, Ztg., 27, 896 (1903).

5 Pfaff: Ibid., 26, 701 (1902).

6 Z. angew. Chem., 18, 93 (1905). 
and the alcohol distilled off, the distillate always contains small quantities of compounds having the characteristic odor of formaldehyde and the power of reducing silver nitrate, but that these compounds are unsaturated bodies of unknown nature.

Other aldehydes can be removed by digestion with resorcin and sulphuric acid, or with aniline and phosphoric acid. Their presence is indicated by the usual aldehyde reactions or by the resorcin test.

Phenols and bases will combine with any formaldehyde that may be made in the course of a test. They must therefore be removed-by distillation from sodium or potassium hydroxide in the case of phenols (this treatment also removes acids)-by distillation from dilute sulphuric acid in the case of bases.

Various compounds such as coloring-matters, etc., can be removed by simple or fractional distillation, by filtration through bone-black with or without previous treatment by lime, or by extracting the alcohol with water and testing the aqueous extract after distillation.

It is best to test only solutions wholly soluble in 2 to 3 parts of water, that will distil between $50^{\circ}$ and $100^{\circ} .{ }^{1}$

Chemists' Club, New York City.

\section{THE ESTIMATION OF FAT IN INFANT AND INVALID FOODS.}

By C. B. Cochran.

Received May 4, I905.

IN THE year I 889 there was published in the Journal of Analytical Chemistry, page $38 \mathrm{r}$, a new process for determining the percentage of fat in milk, cream and skimmed milk. Since that time I have found this process capable of giving very satisfactory results in the determination of fat in condensed milks, infant foods, confections, etc., to which substances the usual methods of fat extraction with ether, or low-boiling gasoline in a Soxhlet apparatus, seem to be inapplicable.

The difficulty of extracting the fat from the dry residue of a diluted, sweetened, condensed milk by the usual fat solvents has been fully appreciated for many years, and other methods have been devised for making this determination.

${ }^{1}$ Mulliken, Scudder: $A m$. Chem. J., 24, 447. The question is here treated entirely from the standpoint of the resorcin test, but the general principles remain the same. 